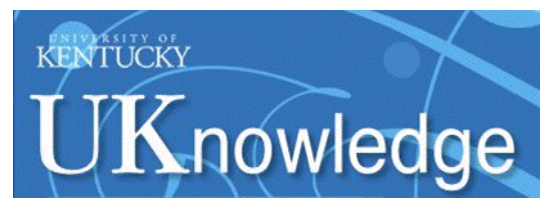

University of Kentucky

UKnowledge

10-1-1984

\title{
Radiation Pressure and the Stability of Broad-Line Region Clouds
}

Gary J. Ferland

University of Kentucky, gary@uky.edu

Moshe Elitzur

University of Kentucky, moshe@pa.uky.edu

Follow this and additional works at: https://uknowledge.uky.edu/physastron_facpub

Part of the Astrophysics and Astronomy Commons, and the Physics Commons

Right click to open a feedback form in a new tab to let us know how this document benefits you.

\section{Repository Citation}

Ferland, Gary J. and Elitzur, Moshe, "Radiation Pressure and the Stability of Broad-Line Region Clouds" (1984). Physics and Astronomy Faculty Publications. 178.

https://uknowledge.uky.edu/physastron_facpub/178

This Article is brought to you for free and open access by the Physics and Astronomy at UKnowledge. It has been accepted for inclusion in Physics and Astronomy Faculty Publications by an authorized administrator of UKnowledge. For more information, please contact UKnowledge@lsv.uky.edu. 


\section{Radiation Pressure and the Stability of Broad-Line Region Clouds}

Digital Object Identifier (DOI)

http://dx.doi.org/10.1086/184354

\section{Notes/Citation Information}

Published in The Astrophysical Journal Letters, v. 285, no. 1, p. L11-L14.

( 1984 . The American Astronomical Society. All rights reserved.

The copyright holder has granted permission for posting the article here. 
THE ASTrophySiCAL Journal, 285:L11-L14, 1984 October 1

(c) 1984. The American Astronomical Society. All rights reserved. Printed in U.S.A.

\title{
RADIATION PRESSURE AND THE STABILITY OF BROAD-LINE REGION CLOUDS
}

\author{
G. J. Ferland and Moshe Elitzur \\ Department of Physics and Astronomy, University of Kentucky \\ Received 1984 March 29; accepted 1984 June 21
}

\begin{abstract}
We discuss two constraints on the existence and stability of quasar emission-line gas. The case of a constant pressure photoionized cloud in pressure equilibrium with a surrounding hot gas is considered. We show that the radiation pressure of the internally generated line emission makes a significant contribution to the total pressure in standard broad-line region models, and that models with either a higher ionization parameter or lower gas density are dominated by radiation pressure and hence unstable. Further, clouds with ionization parameters lower than the standard value cannot be supported by any hot gas which is heated only by the observed radiation field. The result is a strict limit on the range of parameters which permit the existence of the observed emission-line gas. These results offer a natural explanation for the similarities among emission-line spectra of active galactic nuclei.
\end{abstract}

Subject headings: galaxies: nuclei — quasars — radiation mechanisms

\section{INTRODUCTION}

The origin of the line-emitting gas in active galactic nuclei (AGN) has been a long-standing problem of fundamental importance (cf. Davidson 1972; MacAlpine 1972; Blumenthal and Mathews 1975; Krolik, McKee, and Tarter 1981, hereafter KMT). An important constraint on any model is the observation that, to a first approximation, AGN spectra are strikingly similar (Baldwin and Netzer 1978; Davidson and Netzer 1978). A consequence is the deduction that the two free parameters in a photoionization model, density and ionization parameter, must be similar from object to object (Davidson 1977). Why are only some values of these parameters allowed? It is this question we address in this Letter.

\section{TWO CONSTRAINTS ON THE EMITTING GAS}

We follow the basic scenario outlined by, among others, Shields and Oke (1975), Blumenthal and Mathews (1975), Netzer (1980), Weisheit, Shields, and Tarter (1981), Kwan and Krolik (1981, hereafter KK), and KMT in modeling broad-line region (BLR) clouds of $A G N$. In this view the emitting gas has coalesced into a set of small $\left(\delta r \approx 10^{13} \mathrm{~cm}\right)$, cold $\left(T \approx 10^{4}\right.$ $\mathrm{K})$ filaments which are in pressure equilibrium with a surrounding hot $\left(T \approx 10^{8} \mathrm{~K}\right)$ gas. Both hot and cold phases are heated by the radiation field of the central object, although only the cold phase produces significant optical and ultraviolet line emission.

In general, five parameters fully specify a photoionization model. The first three, the shape of the ionizing continuum, the column density, and the chemical composition, are usually set in advance-the first two by comparison with direct observations, and the third by adopting solar-like abundances. In calculations presented below we employ the mixture given by $\mathrm{KK}$, as well as a broken power-law continuum with a steep spectral index of -1.5 (i.e., $f \propto \nu^{-1.5}$ ) for energies between 0.001 and 30 ryd, followed by the flatter spectral index of 0.5 extending to $100 \mathrm{KeV}$ (this continuum gives nearly the same optical-X-ray spectral index as that adopted by $\mathrm{KK}$ and KMT). The column density is $10^{23} \mathrm{~cm}^{-2}$, as deduced from X-ray observations (e.g., Holt et al. 1980). Emission-line intensities are fairly insensitive to these assumptions.

Both the hydrogen density and ionization parameter at the inner face of the cloud are free parameters in this type of calculation (Davidson 1977). A density between $10^{9} \mathrm{~cm}^{-3}$ and $10^{11} \mathrm{~cm}^{-3}$ is set by the absence of forbidden lines such as [O III] 4363, and the presence of C III] 1909 (see Davidson and Netzer 1979). The ionization parameter, a measure of the degree of ionization of the gas, is the last parameter. If $q_{\mathrm{H}}$ is the surface density of ionizing photons $\left(\mathrm{cm}^{-2} \mathrm{~s}^{-1}\right), c$ is the speed of light, and $N_{e}$ is the electron density at the exposed edge of the cloud, then an ionization parameter $U=q_{\mathrm{H}} / N_{e} c$ is the dimensionless ratio of photon to electron densities. Both the ionization and temperature are increasing functions of $U$, and there are homology relations between models with different photon and electron densities, but the same ionization parameter (Tarter, Tucker, and Salpeter 1967). Values of $U$ between 0.01 and 0.1 are deduced from such observational constraints as the C III]/C IV intensity ratio (Davidson 1977; $\mathrm{KK}$ ).

The question why are these values of the density and ionization parameter typical of so many $A G N$, regardless of luminosity, is the one we address here.

\section{a) Radiation Pressure}

The effects of the pressure of trapped line radiation on the stability of the emission regions was addressed first by Williams (1972). Subsequent studies of this effect include work by McKee and Tarter (1975), Mathews (1976), Weymann (1976), and Krolik (1979). All of these seminal studies assumed small column densities (less than $10^{22} \mathrm{~cm}^{-2}$ ), as they were performed before this issue was settled by $\mathrm{KK}$ in favor of larger 
values. The quantitative estimates were also limited essentially to the contribution of $\mathrm{Ly} \alpha$ alone. Most recent detailed models for emission-line regions neglected the contribution to the total pressure of the trapped line radiation.

It is rather simple to show that line radiation pressure can actually contribute significantly to the total pressure for standard values of $U$ and $N$. The radiation pressure inside the cloud due to the emission in a line with frequency $\nu$, line width $\delta \nu$, and at an excitation temperature $T_{x}$ is

$$
P(\nu)=\frac{4 \pi}{3 c} B_{\nu}\left(T_{x}\right) \delta \nu\left(1-\beta_{e}\right),
$$

where $B_{v}$ is the Planck function and $\beta_{e}$ is the escape probability, which can be neglected for optically thick lines. Assuming the line to be thermalized at the gas kinetic temperature $T$, we can calculate the density $n_{p}$ that would make the gas pressure equal to the line radiation pressure. The result is

$$
n_{p}=n_{0} f(x)
$$

where

$$
n_{0}=\frac{8 \pi}{3 \lambda^{3}} \frac{\delta \nu}{\nu}, \quad f(x)=x /[\exp (x)-1], \quad x=h \nu / k T
$$

and $\lambda$ is the wavelength.

Most of the lines of interest have optical depths greater than $10^{4}$, and we set the line width $\delta \nu$ to the frequency at which the optical depth of the Lorentzian profile reaches unity. For the Ly $\alpha$ wavelength of $1216 \AA$, a temperature of $10,000 \mathrm{~K}$, and a fractional linewidth of $\delta \nu / \nu=10^{-3}$, the corresponding density $n_{p}$ is about $4 \times 10^{8} \mathrm{~cm}^{-3}$. This means that at a "typical" BLR cloud density of about $3 \times 10^{9} \mathrm{~cm}^{-3}$, the radiation pressure due to Ly $\alpha$ alone could amount to as much as $10 \%$ of the gas pressure.

Cloud models were calculated using an updated version of the code described by Ferland and Mushotzky (1982). It employs method and assumptions similar to those of KK. A model of the KK "standard cloud" displayed in Figure 1 [i.e., $\log (N)=9.5, \log (U)=-1.5$ ], shows that the Ly $\alpha$ contribution to the radiation pressure is somewhat smaller than the above estimate because the line is subthermalized due to direct photoionization from the hydrogen $n=2$ level. However, the levels of heavier ions cannot be further ionized and can equilibrate with the kinetic temperature if the optical depths are sufficiently large. Many of the strong cooling lines occur at longer wavelengths, closer to the peak of the Planck function, and can contribute significantly to the radiation pressure. For example-about midway in the cloud the temperature is 8000 $\mathrm{K}$ and the total density $1.5 \times 10^{10} \mathrm{~cm}^{-3}$ for a gas pressure of $17 \times 10^{-3} \mathrm{dyn} \mathrm{cm}^{-2}$. At that point the $\mathrm{Mg}$ II 2798 doublet, one of the stronger lines formed in this region, is fully thermalized, and with a fractional linewidth of $1 \times 10^{-3}$, deduced from its optical depth of $2 \times 10^{6}$, it contributes $4 \times 10^{-3}$ dyn $\mathrm{cm}^{-2}$ of radiation pressure. Radiation pressure makes a significant contribution to the total pressure for these standard parameters, and, as we shall see, can actually dominate in many cases.

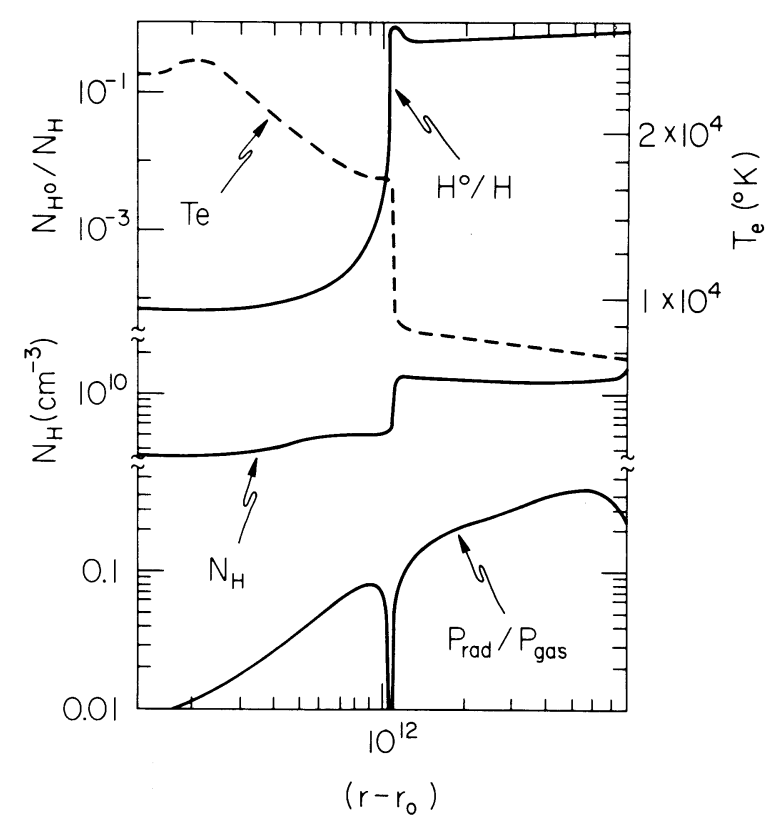

FIG. 1.-A typical broad-line region cloud. Ionization and thermal structure of a typical broad-line region cloud are shown, with parameters similar to those of the standard model of Kwan and Krolik (1980), $U=10^{-1.5}$ and $N_{\mathrm{H}}=10^{9.5} \mathrm{~cm}^{-3}$. See text for more details. Upper curves show the ionization fraction of hydrogen, the electron temperature, and the total hydrogen density. The lower curve shows the ratio $\beta=$ $P($ radiation $) / P($ matter $)$.

Radiation pressure from all strong, optically thick, ultraviolet and optical resonance lines has been added to our calculations. The lower portion of Figure 1 shows the ratio $\beta=P_{\mathrm{rad}} / P_{\text {gas }}$ across this model. The dominant contributors to $\beta$ across the ionized zone are Ly $\alpha$ and C IV 1549, while radiation pressure within the partially ionized zone is caused by $\mathrm{Mg}$ II, Fe II, and C II lines. Radiation pressure is generally negligible near the edge of the cloud because lines are optically thin and the population in the excited states is subthermal. A consequence of the addition of radiation pressure is that the matter density varies less dramatically across the cloud than is typical of models which include only gas pressure in the equation of state. Tests show that the addition of the radiation pressure term changes line intensities by, typically, only $\sim 10 \%$.

To examine the effects of radiation pressure more fully we have computed a grid of models which completely encompass allowed phase space (Fig. 2). Because self-gravity is negligible, the clouds are supported by the external pressure of the confining hot gas, which equals the gas pressure at the skin of the BLR cloud. To be in equilibrium the cloud must maintain a constant pressure throughout. Each model therefore has constant total (gas + radiation) pressure. The figure shows contours of constant pressure (the nearly horizontal lines) as a function of the density and ionization parameter. At low values of $U$ the radiation pressure is negligible, and the gas pressure alone supports the clouds against the external pressure. The near flatness of the curves at this region reflects the fact that for a given chemical composition, the temperature of a gas heated by photoionization is nearly independent of the 


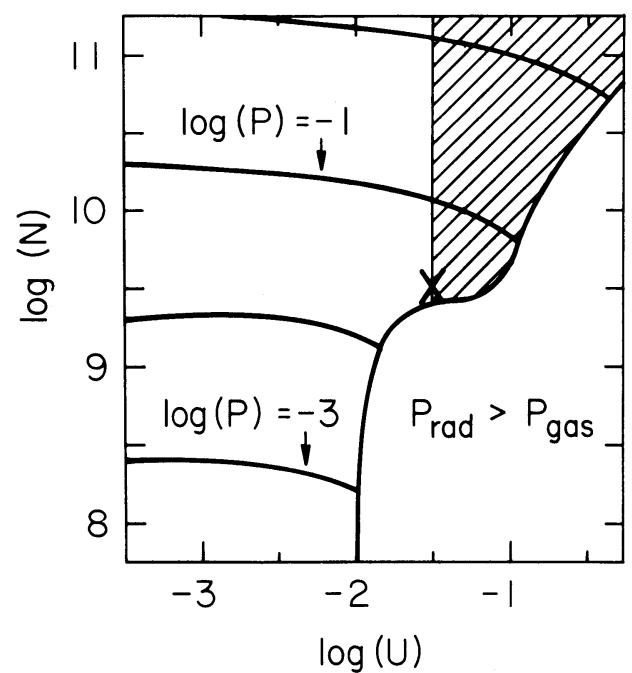

FIG. 2.-The pressure-density-ionization parameter diagram. The axes are the hydrogen density and ionization parameter at the inner face of a constant pressure cloud. Contours of constant pressure are shown as the solid, nearly horizontal, lines. The log of the total pressure $\left(\mathrm{dyn}^{-2}\right)$ is indicated for each contour. Models to the right of the heavy line are unstable to disruption by internally generated radiation pressure. The hatched region shows the portion of the $U-N$ plane where the existence of constant pressure clouds in pressure equilibrium with a hot surrounding medium is possible. The position of the KK "standard" model is denoted by the $X$.

physical parameters. Eventually, for larger values of $U$, the temperature of the gas does increase with $U$, and the isobars turn down.

Radiation pressure first becomes comparable with gas pressure for models with $\log (U) \approx-2$. It is at this ratio of photon to electron density that the energy density in the radiation field becomes comparable with the thermal energy density of the cold gas (Davidson 1977); the radiation field can have little dynamical effect for smaller values of $U$. Whether radiation pressure can become competitive with gas pressure for $\log (U)>-2$ depends mainly on the optical depths of the strong lines which contribute to $P_{\text {rad }}$. Radiation pressure tends to be more important relative to gas pressure for clouds with either low gas densities or high ionization parameters.

As $U$ increases the radiation pressure in the inner regions of the cloud builds up, leading to a decrease in the gas pressure, and particle density, to maintain a constant overall pressure. For sufficiently large values of $U$ the radiation pressure in the deeper regions of the cloud finally exceeds the external pressure, and the gas is driven out. No stable solutions are possible because there is no mechanism to counteract this expulsion. In fact-the decrease in matter density which results from large radiation pressures leads to an increase in the effective ionization parameter at that point in the cloud, which in turn increases both the temperature and radiation pressure, driving even more material out. The result is that only ionization parameters smaller than $U \approx 0.1$ are allowed for typical values of $N$. Accordingly, an upper bound to the ionization parame- ter, set by the constraint that the clouds be stable to disruption by radiation pressure, has been drawn in Figure 2 .

Notice that the requirement that $P_{\text {gas }}$ exceeds $P_{\text {rad }}$ for stable configurations follows directly from the fact that the clouds are supported by an external pressure. It does not apply in the case of, for example, stellar interiors, where the (total) pressure is countered by the varying force of selfgravity.

\section{b) The Confining Medium}

By analogy with the local interstellar medium (cf. Spitzer 1978), it is generally thought that the broad-line region clouds are in pressure equilibrium with a surrounding low-density, hot gas (Blumenthal and Mathews 1975; McCray 1979; KMT; Guilbert, Fabian, and McCray 1983; Lepp et al. 1984). This imposes another constraint on the range of permissible density and ionization parameter (KMT).

Figure 3 shows the temperature and pressure of an optically thin cell of gas with a varying density, exposed to a radiation field with flux density $q_{\mathrm{H}}$ chosen to equal that of a typical BLR cloud $[\log (N)=9.5, \log (U)=-1.5]$. The calculations are basically similar to those of KMT, with minor differences caused by the choice of chemical composition and ionizing continuum. They correspond to a fixed distance from the central source, or a line of constant $U \times N$ in Figure 2, which was chosen to pass through the "standard" cloud parameters.

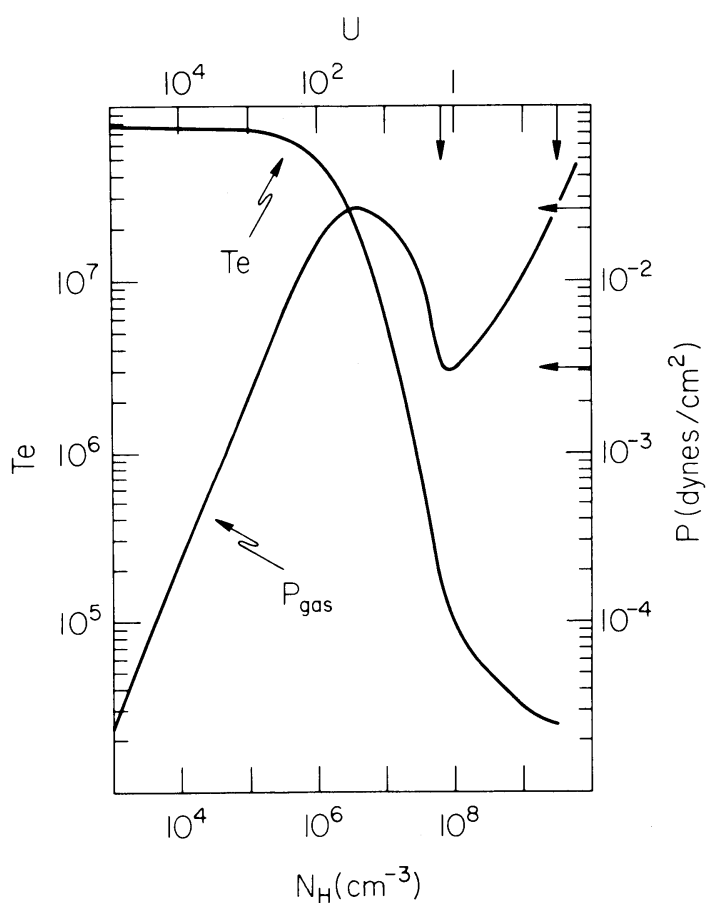

FIG. 3.-Physical conditions at a given radius. Temperature and pressure of optically thin gas with various densities, but at a fixed distance from the central object. These specific calculations are for $\log (U N)=8$, characteristic of the "standard" BLR radius. Three phases can coexist for values of the density near $10^{5}-10^{9.5} \mathrm{~cm}^{-3}$. The range of allowed parameters is indicated by the tick marks. 
Since KMT discuss the implications of this curve at length, we only go over the highlights which affect our discussion. The rapid drop in temperature and pressure near $\log (N)=7$ is caused by the peak in the cooling curve which occurs near $T=10^{5} \mathrm{~K}$ (see Dalgarno and McCray 1972). It is in this region that several gas phases can coexist in pressure equilibrium.

It is evident that for gas at the radius of a standard BLR cloud, the largest hot-phase gas pressure possible is about $0.026 \mathrm{dyn} \mathrm{cm}^{-2}$, a value which is reached at a density of about $3 \times 10^{6} \mathrm{~cm}^{-3}$. It is clear from Figure 3 that no cloud with a density larger than $N=10^{9.5} \mathrm{~cm}^{-3}$ [or equivalently, with an ionization parameter smaller than $\log (U)=-1.5$ ] can be in pressure equilibrium with a hot gas phase at this radius. This constraint, basically the $\Xi_{\min } / \Xi_{\max }$ argument of KMT, imposes a lower bound to the ionization parameter, or, equivalently, an upper bound to the particle density, and shows that large parts of the $U-N$ diagram are inaccessible to pressure-confined clouds. Accordingly, Figure 2 has a line showing the smallest ionization parameter which is consistent with pressure equilibrium with a hot phase. The range of allowed parameters which allow the existence of stable clouds is thus limited to a small portion of parameter space. Note that the lowest value of $U$ consistent with BLR clouds depends also, to some extent, on the precise form of the continuum, as stressed by Guilbert, Fabian, and McCray (1983). The pressure-density law in the crucial portion of Figure 3 is sensitive to both the neon and iron abundances. In summary - although calculations demonstrate the existence of a lower limit, the precise value depends on details such as continuum shape and composition. The point corresponding to the KK standard model lies just within the lower bound of the allowed region.

\section{DISCUSSION}

We have shown that the range of parameters which are consistent with the existence of a constant pressure broad-line region cloud, in pressure equilibrium with a surrounding hotter gas phase, is limited to a small portion of the $U-N$ diagram. Clouds with larger values of the ionization parameter are unstable to disruption by the pressure of the internally generated trapped radiation. A second constraint is imposed by the requirement that it be possible for a hotter gas phase, heated by the same ionizing radiation, to have a pressure at least as great as that of the cold cloud (KMT). This provides a lower bound for the ionization parameter.

These two constraints rule out many values of the density and ionization parameter, and permit only a narrow range of valid solutions. The observed values of $U$ and $N$ tend, however, to favor only a small portion of the allowed region, toward smaller $N$. This point is not addressed in the present study. Another point worth reemphasizing is that all the models presented here have the same column density, which is a crucial parameter in determining the allowed parameters. For example, the standard KK model would disrupt by internal radiation pressure if its column density were increased greatly. All of these points, as well as related issues such as the shape of the boundary in Figure 2, will be discussed in a forthcoming paper (M. Elitzur and G. J. Ferland, in preparation).

Discussions with T. Kallman, R. McCray, H. Netzer, and G. Shields are gratefully acknowledge. We also thank Julian Krolik and Chris McKee for their very useful comments on the manuscript. This work was supported in part by NSF grants AST-8304895 and AST-8305094 to the University of Kentucky.
Baldwin, J. A., and Netzer, H. 1978 Ap. J., 226, 1.

Blumenthal, G. R., and Mathews, W. G. 1975, Ap. J., 198, 517.

Dalgarno, A., and McCray, R. 1972, Ann. Rev. Astr. Ap., 10, 375.

Davidson, K. 1972, Ap. J., 171, 213. .1977, Ap. J., 218, 20.

Davidson, K., and Netzer, H. 1979, Rev. Mod. Phys., 51, 715.

Ferland, G. J., and Mushotzky, R. F. 1982, Ap. J., 262, 564.

Guilbert, P. W., Fabian, A. C., and McCray, R. 1983, Ap. J., 266, 466.

Holt, S. S., Mushotzky, R. F., Becker, R. H., Boldt, E. A., Serlemitsos, P.

Szymkowiak, A. E., and White, N. E. 1980, Ap. J. (Letters), 241, L13.

Krolik, J. H. 1979, Ap. J., 228, 13.

Krolik, J., McKee, C., and Tarter, C. 1981, Ap. J., 249, 422 (KMT).

Kwan, J. 1984, $A p$. J., 283, 70.

Kwan, J., and Krolik, J. 1981, Ap. J., 250, 478 (KK)

Lepp, S., McCray, R., Shull, J., Woods, D., and Kallman, T. 1984 , preprint.

\section{REFERENCES}

MacAlpine, G. M. 1972, Ap. J., 175, 11.

Mathews, W. G. 1976, Ap. J., 207, 351.

McCray, R. 1979, in Active Galactic Nuclei, ed. C. Hazard and S. Mitton, (Cambridge: University of Cambridge Press), p. 227

McKee, C. F., and Tarter, C. B. 1975, Ap. J., 202, 306.

Netzer, H. 1980, Ap. J., 236, 406.

Shields, G. A., and Oke, J. B. 1975, Ap. J., 197, 5.

Spitzer, L. 1978, Physical Processes in the Interstellar Medium (New York: John Wiley and Sons).

Tarter, C. B., Tucker, W., and Salpeter, E. 1967, Ap. J., 156, 943.

Weisheit, J., Shields, G., and Tarter, C. 1981, Ap. J., 245, 406.

Weymann, R. 1976, Ap. J., 208, 286.

Williams, R. E. 1972, Ap. J., 178, 105.

Moshe Elitzur and G. J. Ferland: Department of Physics and Astronomy, University of Kentucky, Lexington, KY 40506-0055 